\title{
COMPLEX RICCATI DIFFERENTIAL EQUATIONS REVISITED
}

\author{
Norbert Steinmetz \\ Technische Universität Dortmund, Institut für Mathematik \\ D-44221 Dortmund, Germany; stein@math.tu-dortmund.de
}

\begin{abstract}
We utilise a new approach via the so-called re-scaling method to derive a thorough theory for polynomial Riccati differential equations in the complex domain.
\end{abstract}

\section{Introduction}

The basic features concerning the value distribution of the solutions to Riccati differential equations

$$
w^{\prime}=a_{0}(z)+a_{1}(z) w+a_{2}(z) w^{2}
$$

with polynomial coefficients are well understood due to the pioneering work of Wittich (see his book [15], Chapter V, pp. 73-80). The solutions are meromorphic in the complex plane, and every non-rational solution has order of growth

$$
\varrho=\limsup _{r \rightarrow \infty} \frac{\log T(r, w)}{\log r}=1+n / 2
$$

mean type, where the non-negative integer $n$ depends on the coefficients $a_{\nu}$ only. The aim of this paper is to refine the results of Wittich and others (Bank [1], Gundersen [5], Hellerstein and Rossi [7, 8]; see also Laine's book [9], Chapter 5) on equation (1) and the associated linear differential equation ( set $a_{2} w=-u^{\prime} / u$ )

$$
u^{\prime \prime}-\left(\frac{a_{2}^{\prime}(z)}{a_{2}(z)}+a_{1}(z)\right) u^{\prime}+a_{0}(z) a_{2}(z) u=0
$$

by a new approach which has been developed earlier to investigate the solutions of Painlevé differential equations (see [12]). By a simple change of variables (retaining the original notation $z, w)$ we obtain

$$
w^{\prime}=a(z)-w^{2}
$$

with

$$
a(z)=z^{n}+O\left(|z|^{n-1}\right) \quad(z \rightarrow \infty) .
$$

Up to finitely many, all poles are simple with residue $1 ; w$ has counting function

$$
n(r, w)=O\left(r^{\varrho}\right) .
$$

Our proofs are solely based on the estimate (4), a new existence proof for asymptotic expansions, and the method of re-scaling.

doi:10.5186/aasfm.2014.3929

2010 Mathematics Subject Classification: Primary 30D30, 30D35, 30D45.

Key words: Riccati differential equation, Stokes sector, Stokes ray, asymptotic series, re-scaling, pole-free sector, Airy equation, Weber-Hermite equation. 


\section{Re-scaling and the distribution of poles}

Throughout the whole paper $w$ denotes any non-rational solution to the Riccati equation $(\mathrm{R})$. For $h \neq 0$ we set

$$
w_{h}(\mathfrak{z})=h^{-n / 2} w\left(h+h^{-n / 2} \mathfrak{z}\right),
$$

where $h^{-n / 2}$ denotes any branch, the same at every occurrence $\left(h^{-n / 2} h^{-n / 2}=h^{-n}\right)$.

Theorem 1. The re-scaled family $\left(w_{h}\right)_{|h|>1}$ is normal in the sense of Montel, and every limit function $\mathfrak{w}=\lim _{h_{n} \rightarrow \infty} w_{h_{n}}$ satisfies the differential equation

$$
\mathfrak{w}^{\prime}=1-\mathfrak{w}^{2} .
$$

We note that the solution $\mathfrak{w}=\operatorname{coth} \mathfrak{z}$ with pole at the origin has the poles $k \pi i$, $k \in \mathbf{Z}$, and no others. Any sequence $\sigma=\left(p_{k}\right)$ satisfying the approximate recursion

$$
p_{k+1}=p_{k}+\omega p_{k}^{-n / 2}+o\left(\left|p_{k}\right|^{-n / 2}\right)
$$

with $\omega= \pm i \pi$ fixed is called a string.

Theorem 2. Let $w$ be any solution to (R). Then the set of poles on $|z|>r_{0}$ consists of finitely many strings of poles. Each string $\sigma$ accumulates at some Stokes ray

$$
s_{\nu}: \arg z=\theta_{\nu}=\frac{(2 \nu+1) \pi}{n+2}
$$

and has counting function

$$
n(r, \sigma)=\frac{r^{\varrho}}{\pi \varrho}+o\left(r^{\varrho}\right) .
$$

Remark. We note that $w$ has Nevanlinna characteristic $T(r, w)=\ell \frac{r^{\varrho}}{\pi \varrho^{2}}+o\left(r^{\varrho}\right)$, where $\ell=\ell(w)$ denotes the number of strings of poles.

\section{Stokes sectors and asymptotic expansions}

The open sectors

$$
S_{\nu}:\left|\arg z-\frac{2 \nu \pi}{n+2}\right|<\frac{\pi}{n+2}
$$

are called Stokes sectors. They are bounded by the Stokes rays $s_{\nu}$ and $s_{\nu-1}$, and will be enumerated as follows:

(a) $0 \leq \nu \leq n+1$ if $n$ is even, and

(b) $-m-1 \leq \nu \leq m+1$ if $n=2 m+1$ is odd.

In the second case $s_{-m-2}=s_{m+1}$ coincides with the negative real axis.

Let $f$ be meromorphic on some sector $S: \phi_{1}<\arg z<\phi_{2}$. Then $f$ is said to have the asymptotic expansion $f \sim \sum_{k=0}^{\infty} c_{k} z^{-k / q}$ for some $q \in \mathbf{N}$, if for every $\delta>0$ and every $n \in \mathbf{N}_{0}$

$$
f(z)-\sum_{k=0}^{n} c_{k} z^{-k / q}=o\left(|z|^{-n / q}\right) \quad(z \rightarrow \infty)
$$

is valid, uniformly on every sub-sector $S(\delta): \phi_{1}+\delta<\arg z<\phi_{2}-\delta$. Obviously, the sector $S$ is 'pole-free' for $f$ in the following sense: to every $\delta>0$ there exists $r(\delta)>0$, such that $f$ has no poles on $S(\delta),|z|>r(\delta)$. It follows from Theorem 2 that the Stokes sectors $S_{\nu}$ are 'pole-free' for every solution to equation (R). By $\sqrt{z}$ 
we denote the branch of the square root with $\operatorname{Re} \sqrt{z}>0$ on $|\arg z|<\pi$, and set $z^{n / 2}=(\sqrt{z})^{n}$ if $n$ is odd.

Theorem 3. The function $z^{-n / 2} w(z)$ has an asymptotic expansion

(a) $\varepsilon+\sum_{k=1}^{\infty} c_{k} z^{-k}$ if $n$ is even, and

(b) $\varepsilon+\sum_{k=1}^{\infty} c_{k} z^{-k / 2}$ if $n$ is odd

on every 'pole-free' sector $S$, with $\varepsilon=\varepsilon(w) \in\{-1,1\}$ and coefficients $c_{k}$ only depending on $\varepsilon$, but neither on $w$ nor the sector $S$. The solution $w$ is uniquely determined by its asymptotic expansion if $S$ contains some sub-sector $S^{\prime}$ such that

$$
\varepsilon \operatorname{Re} z^{\varrho}<0 \text { on } S^{\prime} \text {. }
$$

Remark. In particular, Theorem 3 holds on Stokes sectors $S_{\nu}$ with $\varepsilon=\varepsilon_{\nu}=$ $\varepsilon_{\nu}(w)$. If (8) is valid on $S_{\nu}$, then the corresponding solution is uniquely determined and is denoted by $w_{\nu}$. With every solution $w$ we associate its symbol

(a) $\Sigma=\Sigma(w)=\left[\varepsilon_{0}, \ldots, \varepsilon_{n+1}\right]$ if $n$ is even, and

(b) $\Sigma=\Sigma(w)=\left[\varepsilon_{-m-1}, \ldots, \varepsilon_{m+1}\right]$ if $n=2 m+1$ is odd.

Solutions having the symbol $\Sigma(w)$ with entries $\varepsilon_{\nu}=(-1)^{\nu}$ are called generic. Noting that $(-1)^{\nu} \operatorname{Re} z^{\varrho}>0$ holds on $S_{\nu}$, we obtain from Theorem 3 :

Theorem 4. Any generic solution $w$ has counting function of poles

$$
n(r, w)=\frac{2 r^{\varrho}}{\pi}+o\left(r^{\varrho}\right) .
$$

Theorem 5. Suppose $w$ has symbol $\Sigma$. Then $w$ has

(a) no string of poles asymptotic to the Stokes ray $s_{\nu}$ if $\varepsilon_{\nu}=\varepsilon_{\nu+1}$,

(b) exactly one such string if $(-1)^{\nu}\left(\varepsilon_{\nu}-\varepsilon_{\nu+1}\right)=2$, while

(c) $(-1)^{\nu}\left(\varepsilon_{\nu}-\varepsilon_{\nu+1}\right)=-2$ is impossible.

If $n=2 m+1$ is odd and $\nu=m+1$, the term $\varepsilon_{\nu+1}$ has to be replaced by $-\varepsilon_{-m-1}$. In case (a), $w$ has an asymptotic expansion on $\theta_{\nu-1}<\arg z<\theta_{\nu+1}$. Generic solutions have exactly one string of poles along every Stokes ray, and in any case we have

$$
n(r, w)=\frac{r^{\varrho}}{\pi \varrho} \sum_{\nu}(-1)^{\nu} \epsilon_{\nu}+o\left(r^{\varrho}\right) .
$$

\section{Exceptional solutions}

The non-generic solutions are called exceptional. Exceptional solutions $w_{\nu}$ have the 'false' asymptotics

$$
w_{\nu} \approx-(-1)^{\nu} z^{n / 2} \quad \text { on } S_{\nu}
$$

and are uniquely determined by that condition.

Example 1. The Riccati equation $w^{\prime}=z^{2}+a_{0}-w^{2}$ is closely related to the Weber-Hermite equation

$$
y^{\prime}=y^{2}+2 z y-2-2 \alpha \quad\left(w=-y-z, a_{0}=1+2 \alpha\right) .
$$

There are four exceptional solutions which may be described by their respective symbols $[-\mathbf{1},-1,1,-1],[1, \mathbf{1}, 1,-1],[1,-1,-\mathbf{1},-1]$, and $[1,-1,1, \mathbf{1}]$. The poles are 
distributed along two rays: $|\arg z-\pi|=\frac{\pi}{4},\left|\arg z+\frac{\pi}{2}\right|=\frac{\pi}{4},|\arg z|=\frac{\pi}{4}$, and $\left|\arg z-\frac{\pi}{2}\right|=\frac{\pi}{4}$, respectively.

Example 2. The Riccati equation $w^{\prime}=z+a_{0}-w^{2}$ is closely related to the Airy equation $y^{\prime}=z / 2+y^{2}$. It has three exceptional solutions with symbols $[-1,-\mathbf{1},-1]$, $[\mathbf{1}, 1,-1]$, and $[-1,1, \mathbf{1}]$, and strings of poles asymptotic to (actually: on) $\arg z=\pi$, $\arg z=\pi / 3$, and $\arg z=-\pi / 3$, respectively.

Theorem 6. To every Stokes sector $S_{\nu}$ there exists a unique exceptional solution $w_{\nu}$. It has the asymptotic expansion (9) also on the Stokes sectors adjacent to $S_{\nu}$, and no strings of poles along the Stokes rays that form the boundary of $S_{\nu}$. The number $d_{\nu}=n-\ell_{\nu}$, where $\ell_{\nu}$ denotes the number of strings of poles of $w_{\nu}$, is even.

Remark. The exceptional solutions $w_{\nu}$ correspond to those solutions to the linear differential equation $y^{\prime \prime}=a(z) y$ that are sub-dominant on $S_{\nu} ; y_{\nu}=\exp \int w(z) d z$ is called sub-dominant on $S_{\nu}$, if $y_{\nu}$ tends to zero exponentially as $z \rightarrow \infty$ on $S_{\nu}$.

Example 3. Gundersen and Steinbart [6] considered the linear differential equation $f^{\prime \prime}-z^{n} f=0$. They proved among others that certain contour integrals

$$
f_{\nu}(z)=\frac{1}{2 \pi i} \int_{C_{\nu}} e^{P(z, w)} d w
$$

represent solutions having no zeros along given Stokes rays $s_{\nu-1}$ and $s_{\nu}$. These solutions give rise to exceptional solutions $w_{\nu}=f_{\nu}^{\prime} / f_{\nu}$ to the special Riccati equation $w^{\prime}=z^{n}-w^{2}$, which is invariant under the transformations $w(z) \mapsto \eta w(\eta z), \eta^{n+2}=$ 1. There are exactly two solutions that are invariant under these transformations, namely those which either have a pole or else a zero at the origin. These solutions are generic, hence there are $n+2$ mutually distinct exceptional solutions. They are obtained from a single one, $w_{0}$, say, by rotating the plane:

$$
w_{\nu}(z)=e^{\frac{2 \nu \pi i}{n+2}} w_{0}\left(e^{\frac{2 \nu \pi i}{n+2}} z\right)
$$

$w_{\nu}$ has a single string of poles along every Stokes ray $s_{\mu}$ except those that bound the Stokes sector $S_{\nu}$.

In the general case $(\mathrm{R})$ the solutions $w_{\nu}$ need not be mutually distinct.

Example 4. The eigenvalue problem $f^{\prime \prime}+\left(z^{4}-\lambda\right) f=0, f \in L^{2}(\mathbf{R})$, has infinitely many solutions $\left(\lambda_{k}, f_{k}\right)\left(0<\lambda_{k} \rightarrow \infty\right)$, see Titchmarsh [13]. The eigenfunctions $f_{k}$ have only finitely many non-real zeros. For every eigenpair $(\lambda, f)=\left(\lambda_{k}, f_{k}\right)$, $u(z)=f\left(e^{-i \pi / 6} z\right)$ satisfies $u^{\prime \prime}-\left(z^{4}+e^{-i \pi / 3} \lambda\right) u=0$, and $w=u^{\prime} / u$ solves

$$
w^{\prime}=z^{4}+e^{-i \pi / 3} \lambda-w^{2} .
$$

Up to finitely many the poles of the exceptional solution $w=w_{2}=w_{5}$ belong to the rays $\arg z=\frac{\pi}{6}$ and $\arg z=\frac{7}{6} \pi$, hence $w$ has the symbol $[1,-1,-\mathbf{1},-1,1, \mathbf{1}]$.

Example 5. Eremenko and Gabrielov [2] considered the linear equation

$$
y^{\prime \prime}-\left(z^{3}-a z+\lambda\right) y=0 .
$$

For certain real parameters $a$ and $\lambda$ it has solutions with infinitely many zeros, only finitely many of them are non-real or real and positive. Thus $w^{\prime}=z^{3}-a z+\lambda-w^{2}$ has a solution $w$ with symbol $[1, \mathbf{1}, 1, \mathbf{1}, 1]$, hence $w=w_{1}=w_{-1}$, and mutually distinct solutions $w_{0}, w_{-2}$, and $w_{2}$ with symbols $[1,-1,-\mathbf{1},-1,1],[-\mathbf{1},-1,1,-1,1]$, and $[1,-1,1,-1,-\mathbf{1}]$, respectively, each having three strings of poles. 


\section{Poles close to a single line}

Several papers (Eremenko and Merenkov [3], Eremenko and Gabrielov [2], Gundersen [4, 5], Shin [11]) are devoted to the question whether or not the linear differential equation

$$
y^{\prime \prime}-P(z) y=0 \quad\left(P(z)=a_{n} z^{n}+\cdots \text { a polynomial of degree } n,\left|a_{n}\right|=1\right)
$$

has solutions with all but finitely many zeros on the real axis. From Theorem 5 we obtain (see also $[3,4]$ ):

Theorem 7. Suppose that equation (10) has a solution whose zeros are asymptotic to the real axis. Then the following is true:

If $n$ is even, then either

- y has only finitely many zeros, or else

- $n \equiv 0 \quad \bmod 4, a_{n}=-1, y$ has exactly one string of zeros asymptotic to the negative and positive real axis, and $y^{\prime} / y \approx \mp i z^{n / 2}$ holds on the upper and lower half-plane, respectively.

If $n=2 m+1$ is odd, then either

- $a_{n}=1, y$ has exactly one string of poles asymptotic to the negative real axis with asymtotics $y^{\prime} / y \approx(-1)^{m+1} z^{n / 2}$ on $|\arg z|<\pi$, or else

- $a_{n}=-1, y$ has exactly one string of poles asymptotic to the positive real axis with asymtotics $y^{\prime} / y \approx(-1)^{m+1}(-z)^{n / 2}$ on $|\arg (-z)|<\pi$.

If $P$ is real, then in each case all but finitely many zeros are real and $y$ is a (multiple of a) real entire function.

\section{The Schwarzian derivative}

In [10] Nevanlinna considered the locally univalent meromorphic functions $f$ of finite order. They are characterised by the fact that their Schwarzian derivative $S_{f}=\left(f^{\prime \prime} / f^{\prime}\right)^{\prime}-\frac{1}{2}\left(f^{\prime \prime} / f^{\prime}\right)^{2}$ is a polynomial $2 P$, say. Moreover, $f$ is the quotient $y(z ; 0) / y(z ; \infty)$ of two linearly independent solutions to the linear differential equation

$$
y^{\prime \prime}+P(z) y=0,
$$

which is equivalent to the Riccati equation $w^{\prime}=-P(z)-w^{2}$ via $w=y^{\prime} / y$. The generic solutions have counting function of poles and Nevanlinna characteristic $T(r, w)$ $\sim C r^{\varrho}$ with $\varrho=1+\frac{1}{2} \operatorname{deg} P ; C>0$ is some known constant. Every exceptional solution $w_{\nu}$, however, has counting function and Nevanlinna characteristic $T\left(r, w_{\nu}\right) \sim C \frac{n+2-2 d_{\nu}}{n+2} r^{\varrho}$, where $d_{\nu}$ is some positive integer such that $\sum_{\nu} d_{\nu}=n+2$. Since the zeros of $f-a$ are the same as the zeros of $y(z ; a)=y(z ; 0)-a y(z ; \infty)$, hence coincide with the poles of $w(z ; a)=y^{\prime}(z ; a) / y(z ; a)$, it follows that $f$ has Nevanlinna deficiencies $\delta\left(a_{\nu}\right)=\frac{2 d_{\nu}}{n+2}\left(w_{\nu}(z)=w\left(z ; a_{\nu}\right)\right)$ with $\sum_{\nu} \delta\left(a_{\nu}\right)=2$.

\section{Proof of Theorem 1 and Theorem 2}

Proof of Theorem 1. From

$$
w_{h}^{\prime}(\mathfrak{z})=h^{-n} a\left(h+h^{-n / 2} \mathfrak{z}\right)+w_{h}(\mathfrak{z})^{2}
$$

and $z^{-n} a(z) \rightarrow 1$ as $z \rightarrow \infty$ it follows that

$$
\left|w_{h}^{\prime}(\mathfrak{z})\right| \leq 2+\left|w_{h}(\mathfrak{z})\right|^{2}
$$


holds on $|\mathfrak{z}|<R,|h|>\eta_{R}$. Thus the family $\left(w_{h}^{\sharp}\right)_{|h| \geq 1}$ of spherical derivatives

$$
w_{h}^{\sharp}=\frac{\left|w_{h}^{\prime}\right|}{1+\left|w_{h}\right|^{2}}
$$

is bounded on $|\mathfrak{z}|<R$ by $M(R)=\sup \left\{w_{h}^{\sharp}(\mathfrak{z}):|\mathfrak{z}|<R, 1<|h|<\eta_{R}\right\}+2$, say. The limit function $\mathfrak{w}=\lim _{h_{k} \rightarrow \infty} w_{h_{k}} \equiv \infty$ does not occur since otherwise $u_{h_{k}}=1 / w_{h_{k}}$ would tend to zero, this contradicting $u_{h_{k}}^{\prime}=1-h_{k}^{-n} a\left(h_{k}+h_{k}^{-n / 2} \mathfrak{z}\right) u_{h_{k}}^{2} \rightarrow 1$. Thus every limit function $\mathfrak{w}$ satisfies (5) outside the set $\mathfrak{P}$ of poles of $\mathfrak{w}$.

Proof of Theorem 2. From Theorem 1 and Hurwitz' Theorem it follows that given $\epsilon>0$ and $R>0$ there exists some $r_{0}>0$, such that the disc

$$
\triangle_{R}(p)=\left\{z:|z-p|<R|p|^{-n / 2}\right\}
$$

about any pole $p$ with $|p|>r_{0}$ contains the poles $\tilde{p}_{k}$ with

$$
\left|\tilde{p}_{k}-\left(p+k \pi i p^{-n / 2}\right)\right|<\epsilon|p|^{-n / 2} \quad\left(-k_{1}(p) \leq k \leq k_{2}(p)\right),
$$

and no others; the numbers $k_{1}$ and $k_{2}$ are bounded by a number only depending on $R$ (for example, $k_{1}=k_{2}=318$ if $R=1000$ and $r_{0}$ is sufficiently large). Thus up to finitely many every pole is contained in a unique string of poles $\left(p_{k}\right)$ satisfying (6). Then $z_{k}=p_{k}^{\varrho}(\varrho=n / 2+1)$ satisfies

$$
z_{k+1}=z_{k}+\omega \varrho+o(1)
$$

with $\omega= \pm \pi i$ fixed, hence $z_{k}=\omega \varrho k+o(k), p_{k}=(\omega \varrho k)^{1 / \varrho}(1+o(1))$, and

$$
\frac{n+2}{2} \arg p_{k}=\arg \omega+o(1)= \pm \frac{\pi}{2}+o(1) \quad \bmod 2 \pi
$$

that is, $\arg p_{k}=\theta_{\nu}+o(1)=\frac{2 \nu \pi+1}{n+2}+o(1)$ holds for some $\nu$. The counting function of $\sigma$ equals $n(r, \sigma)=\frac{r^{\varrho}}{\pi \varrho}+o\left(r^{\varrho}\right)$, and from $n(r, w)=O\left(r^{\varrho}\right)$ it follows that there are only finitely many strings of poles.

\section{Proof of Theorem 3}

Let $w$ be any solution to $(\mathrm{R})$ and $S:\left|\arg z-\phi_{0}\right|<\eta$ any sector that is "polefree' for $w$. From Theorem 1 then it follows that $w(z) z^{-n / 2}$ tends to either +1 or else -1 as $z \rightarrow \infty$; the convergence to +1 , say, is uniform on each closed subsector $S(\delta):\left|\arg z-\phi_{0}\right| \leq \eta-\delta$ (take any sequence $h_{k} \rightarrow \infty$ in $S(\delta)$ such that $\lim _{h_{k} \rightarrow \infty}\left|w\left(h_{k}\right) h_{k}^{-n / 2}-1\right|=\limsup _{z \rightarrow \infty}\left|w(z) z^{-n / 2}-1\right|$ on $\left.S(\delta)\right)$. If $n=2 m$ is even we set $v(z)=z^{-m} w(z)$ to obtain

$$
z^{-m} v^{\prime}+m z^{-m-1} v=a(z) z^{-2 m}-v^{2} .
$$

If, however, $n=2 m+1$ is odd we set $v(z)=z^{-n} w\left(z^{2}\right)$ to obtain

$$
z^{-n-1} v^{\prime}+n z^{-n-2} v=2 a\left(z^{2}\right) z^{-2 n}-2 v^{2} .
$$

From (11) resp. (12) and the fact that $v(z) \rightarrow \pm 1$ on some sector $S$ we have to conclude $v \sim \pm 1+\sum_{k=1}^{\infty} c_{k} z^{-k}$ on $S$. For definiteness we will consider equation (11) with $v(z) \rightarrow 1$ on $S$. If we assume that

$$
v(z)=1+\sum_{k=1}^{n} c_{k} z^{-k}+o\left(|z|^{-n}\right)=\psi_{n}(z)+o\left(|z|^{-n}\right)
$$


has already been proved (this is true for $n=0$ ) we obtain from

$$
v^{\prime}(z)=\psi_{n}^{\prime}(z)+o\left(|z|^{-n-1}\right)
$$

and (11)

$$
a(z) z^{-2 m}-v^{2}=z^{-m} \psi_{n}^{\prime}(z)+m z^{-m-1} \psi_{n}(z)+o\left(|z|^{-n-m-1}\right) .
$$

The algebraic equation

$$
a(z) z^{-2 m}-y^{2}=z^{-m} \psi_{n}^{\prime}(z)+m z^{-m-1} \psi_{n}(z)
$$

has a unique solution $y=1+\sum_{k=1}^{\infty} c_{k}^{\prime} z^{-k}$ about $z=\infty$, and from $v+y=2+o(1)$ and $(v-y)(v+y)=v^{2}-y^{2}=o\left(|z|^{-n-m-1}\right)$ it follows that

$$
v=y+o\left(|z|^{-n-m-1}\right)=1+\sum_{k=1}^{n+1} c_{k}^{\prime} z^{-k}+o\left(|z|^{-n-1}\right)=\psi_{n+1}(z)+o\left(|z|^{-n-1}\right) .
$$

It is obvious that $c_{k}=c_{k}^{\prime}$ holds for $0 \leq k \leq n$, and this proves the existence part. The proof is the same in all other cases.

To prove the uniqueness part of Theorem 3 we assume that $w_{1}$ and $w_{2}$ have the same asymptotic expansion on the sector $S$. Then $u=w_{1}-w_{2}$ solves

$$
u^{\prime}=-\left(w_{1}(z)+w_{2}(z)\right) u=-2 \varepsilon z^{n / 2}\left(1+O\left(|z|^{-\frac{1}{2}}\right)\right) u,
$$

hence $u=C \exp \left(-\frac{2 \varepsilon}{\varrho} z^{\varrho}+O\left(|z|^{\varrho-\frac{1}{2}}\right)\right)$ holds. Our hypothesis $\varepsilon \operatorname{Re} z^{\varrho}<0$ and $u \rightarrow 0$ on $S^{\prime} \subset S$ then gives $u=C=0$, and this proves Theorem 3 completely.

\section{Proof of Theorem 5}

Since all but finitely many poles of $w$ are simple with residue 1 , the Residue Theorem gives

$$
n(r, w)=\frac{1}{2 \pi i} \int_{\Gamma_{r}} w(z) d z+O(1)
$$

where the simple closed curve $\Gamma_{r}$ is obtained from the circle $C_{r}:|z|=r$ by replacing the intersection of $C_{r}$ with any disc $\triangle_{\epsilon}(p)=\left\{z:|z-p|<\epsilon|p|^{-n / 2}\right\}(\epsilon>0$ sufficiently small, $p$ any pole of $w)$ by an appropriate sub-arc of $\partial \triangle_{\epsilon}(p)$. From $w=O\left(|z|^{n / 2}\right)=$ $O\left(|z|^{\varrho-1}\right)$ on $\Gamma_{r}$ (this following from the normality of the family $w_{h}(\mathfrak{z})=h^{-n / 2} w(h+$ $\left.\left.h^{-n / 2} \mathfrak{z}\right)\right)$ and the fact that $\Gamma_{r} \cap\left\{z:\left|\arg z-\theta_{\nu}\right|<\delta\right\}$ has length at most $2 \pi \delta r$ as $\delta \rightarrow 0$, it follows that the contribution of the Stokes sector $S_{\nu}$ to the counting function of poles equals

$$
(-1)^{\nu} \varepsilon_{\nu} \frac{r^{\varrho}}{\pi \varrho}+o\left(r^{\varrho}\right) \quad(\varrho=n / 2+1) .
$$

In particular, $w$ has $\sum_{\nu}(-1)^{\nu} \varepsilon_{\nu}$ strings of poles. Integrating $w$ along the line segment $\sigma$ from $r_{0} e^{i\left(\theta_{\nu}-\delta\right)}\left(\delta>0\right.$ small, $r_{0}>0$ large $)$ to $r e^{i\left(\theta_{\nu}-\delta\right)}$ gives

$$
\frac{1}{2 \pi i} \int_{\sigma} w(z) d z=\frac{\varepsilon_{\nu}}{2 \pi i \varrho} r^{\varrho} e^{i \varrho\left(\theta_{\nu}-\delta\right)}+o\left(r^{\varrho}\right)=(-1)^{\nu} \frac{\varepsilon_{\nu}}{2 \pi \varrho} e^{-i \varrho \delta} r^{\varrho}+o\left(r^{\varrho}\right) .
$$

Thus, if $\gamma_{r}^{\nu}$ denotes the simple closed curve which consists of the line segment $\sigma$, the part of $\Gamma_{r}$ from $r e^{i\left(\theta_{\nu}-\delta\right)}$ to $r e^{i\left(\theta_{\nu}+\delta\right)}$, the line segment from $r e^{i\left(\theta_{\nu}+\delta\right)}$ to $r_{0} e^{i\left(\theta_{\nu}+\delta\right)}$, and the circular arc on $|z|=r_{0}$ from $r_{0} e^{i\left(\theta_{\nu}+\delta\right)}$ to $r_{0} e^{i\left(\theta_{\nu}-\delta\right)}$ we obtain

$$
\frac{1}{2 \pi i} \int_{\gamma_{r}^{\nu}} w(z) d z=(-1)^{\nu} \frac{r^{\varrho}}{2 \pi \varrho}\left[\varepsilon_{\nu}-\varepsilon_{\nu+1}\right]+O\left(\delta r^{\varrho}\right)+o\left(r^{\varrho}\right)
$$


$(r \rightarrow \infty, \delta \rightarrow 0)$. Now the integral on the left hand side equals the number of poles inside $\gamma_{r}^{\nu}$, while $(-1)^{\nu} \frac{1}{2}\left[\varepsilon_{\nu}-\varepsilon_{\nu+1}\right]$ coincides with the number of strings of poles along the Stokes ray $s_{\nu}$ : $\arg z=\theta_{\nu}$. From this the assertions (a), (b), and (c) in Theorem 5 immediately follow.

\section{Proof of Theorem 6}

It is easily seen that equation (11) resp. (12), written as

$$
z^{-q} v^{\prime}=f(z, v) \quad(q=m \text { resp. } q=n+1)
$$

has a formal solution $\varepsilon_{\nu}+\sum_{\nu=1}^{\infty} c_{\nu} z^{-\nu}$ with $\varepsilon_{\nu}=-(-1)^{\nu}$. Since $\lim _{z \rightarrow \infty} f_{v}\left(z, \varepsilon_{\nu}\right)=$ $-2 \varepsilon_{\nu} \neq 0$, Theorem 12.1 in Wasow's monograph [14] applies to the corresponding equation for $v-\varepsilon_{\nu}$. Hence to every sector $\left|\arg z-\theta_{0}\right|<\frac{\pi}{2 q+2}$ there exists a solution to equation (14) with asymptotic expansion $v \sim \varepsilon_{\nu}+\sum_{\nu=1}^{\infty} c_{\nu} z^{-\nu}$. In particular, for every $\nu$ we obtain a (unique) solution $w=w_{\nu}$ to $(\mathrm{R})$ with the desired asymptotic expansion (9) on the Stokes sector $S_{\nu}$.

\section{Proof of Theorem 7}

If $y(z)=P_{1}(z) e^{P_{2}(z)}$ has only finitely many zeros, then $n=2 \operatorname{deg} P_{2}-2$ is even, and not much more can be said (of course, $P$ can be computed explicitly from $P_{1}$ and $P_{2}$ ). From now on we assume that $y$ has infinitely many zeros. The change of variables $w(z)=\eta y^{\prime}(\eta z) / y(\eta z)$ with $\eta^{n+2} a_{n}=1$ transforms equation (10) into equation (R) with $a(z)=\eta^{2} P(\eta z)=z^{n}+\cdots$, hence the question whether or not there are solutions $y$ to (10) having infinitely many zeros, 'most' of them close to the real axis is transformed into the question for solutions $w$ to $(\mathrm{R})$ having just one string of poles asymptotic to some Stokes ray $s_{\nu}$ : $\arg z=\theta_{\nu}=\frac{(2 \nu+1) \pi}{n+2}$ if $n$ is odd, and asymptotic to the Stokes rays $s_{\nu}$ and $s_{\nu+m}$ if $n=2 m$ is even, respectively. This yields $\bar{\eta}= \pm e^{i \theta_{\nu}}$ up to an arbitrary root of unity of order $n+2$, and we are free to choose $\eta=e^{-i \frac{\pi}{n+2}}$ and $\nu=0$ if $n$ is even, and $\eta= \pm 1$ and $\nu=m+1$ if $n=2 m+1$ is odd. In the first case we obtain $a_{n}=-1$, and from Theorem 5 it follows that $\epsilon_{0}-\epsilon_{1}=2$ and $(-1)^{m+1}\left(\epsilon_{m+1}-\epsilon_{m+2}\right)=2$, hence $\epsilon_{0}=1$ and $\epsilon_{1}=-1$, this implying $\epsilon_{2}=\cdots=\epsilon_{m+1}=\epsilon_{1}=-1, \epsilon_{m+2}=\cdots=\epsilon_{2 m+1}=\epsilon_{0}=1, m=2 k$ and $n=4 k$. This proves the first part of Theorem 6 .

In the second case we have $a_{n}=+1$ and $a_{n}=-1$ with zeros asymptotic to the negative and positive real axis, respectively, and asymptotic expansions $y^{\prime} / y \approx$ $(-1)^{m+1} z^{n / 2}$ on $|\arg z|<\pi$ resp. $y^{\prime} \mid y \approx(-1)^{m+1}(-z)^{n / 2}$ on $|\arg (-z)|<\pi$ (note that $z^{n / 2}$ means $\left.(\sqrt{z})^{n}\right)$.

Now $y$ is uniquely determined up to a constant factor. Thus if $P$ is a real polynomial, then the zeros of $y^{*}(z)=\overline{y(\bar{z})}$ are also asymptotic to the real axis, hence $y$ and $y^{*}$ are linearly dependent, and $y$ is a multiple of a real function with all but finitely many zeros real.

\section{References}

[1] BANk, S.: A note on the zeros of solutions of $w^{\prime \prime}+P(z) w=0$, where $P$ is a polynomial. Appl. Anal. 25, 1988, 29-41.

[2] Eremenko, A., and A. Gabrielov: Singular pertubation of polynomial potentials with application to PT-symmetric families. - Mosc. Math. J. 11, 2011, 473-503. 
[3] Eremenko, A., and S. Merenkov: Nevanlinna functions with real zeros. - Illinois J. Math. 49, 2005, 1093-1110.

[4] Gundersen, G.: On the real zeros os solutions of $f^{\prime \prime}+A(z) f=0$, where $A$ is entire. - Ann. Acad. Sci. Fenn. Ser. A I Math. 11, 1986, 275-294.

[5] Gundersen, G.: Solutions of $f^{\prime \prime}+P(z) f=0$ that have almost all real zeros. - Ann. Acad. Sci. Fenn. Math. 26, 2001, 483-488.

[6] Gundersen, G., and E. Steinbart: A generalization of the Airy integral for $f^{\prime \prime}-z^{n} f=0$. - Trans. Amer. Math. Soc. 337, 1993, 737-755.

[7] Hellerstein, S., and J. Rossi: Zeros of meromorphic solutions of second-order differential equations. - Math. Z. 192, 1986, 603-612.

[8] Hellerstein, S., and J. Rossi: On the distribution of zeros of solutions of second-order differential equations. - Complex Var. Theory Appl. 13, 1989, 99-109.

[9] Laine, I.: Nevanlinna theory and complex differential equations. - W. de Gruyter, 1993.

[10] Nevanlinna, R.: Über Riemannsche Flächen mit endlich vielen Windungspunkten - Acta. Math. 58, 1932, 295-273.

[11] Shin, K.: New polynomials $P$ for which $f^{\prime \prime}+P(z) f=0$ has a solution with almost all real zeros. - Ann. Acad. Sci. Fenn. Math. 27, 2002, 491-498.

[12] Steinmetz, N.: Sub-normal solutions to Painlevé's second differential equation. - Bull. London Math. Soc. 45, 2013, 225-235.

[13] Titchmarsh, E. C.: Eigenfunction expansions associated with second-order differential equations, Part I, second edition. - Oxford Univ. Press, London, 1962.

[14] Wasow, W.: Asymptotic expansions for ordinary differential equations. - J. Wiley \& Sons, 1965.

[15] Wiтtich, H.: Neuere Untersuchungen über eindeutige analytische Funktionen. - Springer, 1968.

Received 30 January 2013 • Accepted 20 December 2013 